Published in final edited form as:

J Org Chem. 2006 February 17; 71(4): 1430-1435.

\title{
C-Metalated Nitriles: Electrophile-Dependent Alkylations and
}

\section{Acylations}

\author{
Fraser F. Fleming ${ }^{*}$, Zhiyu Zhang, Guoqing Wei, and Omar W. Steward \\ Department of Chemistry and Biochemistry, Duquesne University, Pittsburgh, Pennsylvania \\ 15282-1530.
}

\section{Abstract}

Sequential carbonyl addition-conjugate addition of Grignard reagents to 3-oxocyclohex-1-ene-1carbonitrile generates $C$-magnesiated nitriles whose alkylation stereoselectivities intimately depend on the nature of the electrophile. Alkylating these $C$-magnesiated nitriles with alkyl halides, sulfonates, and unstrained ketones occur with retention of the $\mathrm{C}-\mathrm{Mg}$ configuration, whereas aldehyde and acyl cyanide acylations proceed with inversion of stereochemistry. Mechanistic probes indicate that the stereoselectivity is controlled by stereoelectronic effects for most electrophiles except allylic, benzylic, and cyclopropyl halides where single electron transfer processes intervene. Screening numerous alkylations of $C$-magnesiated nitriles with a diverse range of electrophiles reveals the reaction scope and delineates the fundamental stereoelectronic effects responsible for the highly unusual electrophile-dependent alkylations.

\section{Introduction}

Metalated nitriles are powerful nucleophiles that are broadly effective in a diverse range of alkylations. ${ }^{1}$ The exceptional nucleophilicity stems from the inductively stabilized ${ }^{2}$ negative charge density localized on the formally anionic carbon. Inductive stabilization ${ }^{2}$ of metalated nitriles, rather than resonance delocalization, is conspicuous from nucleophilicity trends, ${ }^{3}$ acidity measurements, ${ }^{4}$ and NMR analyses, ${ }^{5}$ and is clearly manifest in the bond distances of metalated nitriles in the solid state. ${ }^{6}$ Particularly significant is the $\mathrm{C} \equiv \mathrm{N}$ bond length (1.14-1.20 $\AA$, Figure 1) of metalated nitriles, which is virtually identical to the $\mathrm{C} \equiv \mathrm{N}$ bond length of neutral nitriles $(1.14 \AA) .{ }^{7}$ Correspondingly, the C-CN bond length (1.36-1.45 $\AA$ ) is appreciably contracted relative to a $\mathrm{C}-\mathrm{C}$ single bond, reflecting the electrostatic attraction between the negatively charged 'carbanion' and the strongly ${ }^{2}$ electron withdrawing nitrile group.

Inductive stabilization of metalated nitriles creates two potential metal coordination sites: at the nitrile nitrogen, and at the adjacent anionic carbon (Figure 1, compare 3 and 4). Extensive solid state ${ }^{6}$ and solution ${ }^{8}$ analyses reveal that lithiated nitriles exhibit a distinct preference for $N$-coordination with a planar geometry at the formally anionic carbon (1, Figure 1). ${ }^{9} \mathrm{In}$ contrast, the sodiated nitrile $\mathbf{2}$ is $\mathrm{N}$-metalated and partially pyramidal, typifying the range of solid state structures observed for metalated nitriles. ${ }^{10}$ Metalated nitriles with transition metal counter ions show an almost equal propensity for $C$ - and $N$-coordination ${ }^{11}$ with several $N$ metalated nitriles having ruthenium ${ }^{12}$ or palladium ${ }^{13}$ counter ions, such as 3 , being converted to their thermodynamically more stable $C$-metalated counterparts $\mathbf{4}$, upon heating. The geometry and coordination of synthetically valuable magnesiated ${ }^{14}$ and zincated nitriles 15

flemingf@duq.edu

Supporting Information Available. Experimental procedures, ${ }^{1} \mathrm{H}$ NMR, and ${ }^{13} \mathrm{C}$ NMR spectra for all new compounds and CIF and ORTEP's for all structures assigned by $\mathrm{x}$-ray crystallography. This material is available free of charge via the Internet at http://pubs.acs.org. 
remains to be determined by crystallography, although NMR and IR analyses indicate $C$ metalation in both cases. ${ }^{14-16}$

Distinctly different regio- and stereoselectivity preferences emanate from $\mathrm{N}$ - and $\mathrm{C}$-metalated nitriles. For example, intercepting the putative ${ }^{8} N$-lithiated nitrile $6 \mathbf{a}$ with propargyl bromide affords alkynenitrile 7a through an $\mathrm{S}_{\mathrm{N}} 2$ displacement (Scheme 1), whereas alkylation of the analogous $C$ - cuprated nitrile $8 \mathbf{a}$ with propargyl bromide gives the $S_{N} 2^{\prime}$ allenylnitrile $7 \mathbf{b}$. ${ }^{14 a}$ Similarly, alkylations of the $N$-lithiated, conformationally locked nitrile $\mathbf{6 b}$ are only modestly diastereoselective, with preferential equatorial methylation affording $7 \mathbf{c}$ in a $2.8: 1$ ratio $(86 \%)$. ${ }^{17}$ In contrast, alkylation of the putative $C$-magnesiated nitrile $\mathbf{8 b}$ affords $7 \mathbf{c}$ exclusively, under otherwise identical conditions (Scheme 1). ${ }^{14 \mathrm{~b}}$

The excellent alkylation stereoselectivities of $C$-metalated nitriles highlights the potential of configurationally stable, chiral, $C$-metalated nitriles. Experimentally, overcoming the difficulty of synthesizing $C$-magnesiated nitriles is possible by employing internal coordination to favor formation of pyramidal, $C$-magnesiated nitriles. ${ }^{18}$ Remarkably, the resulting $C$ magnesiated nitriles exhibit electrophile-dependent alkylation stereoselectivities previously unprecedented in alkylations of metalated nitriles. ${ }^{19}$ Comprehensively surveying the alkylations of cyclic $C$-magnesiated nitriles establishes key electrophile-dependent alkylation trends, reveals the scope of the reaction, and delineates the fundamental stereoelectronic effects responsible for the unusual stereoselectivity preferences.

\section{Results and Discussion}

Synthesis of an asymmetric, $C$-magnesiated nitrile was probed by deprotonating a cyclohexanecarbonitrile capable of strong internal coordination. Direct formation of magnesiated nitriles is challenging since magnesium amides do not effectively deprotonate aliphatic nitriles, ${ }^{20}$ whereas Grignard reagents typically ${ }^{21}$ add to, as well as deprotonate, unactivated nitriles. ${ }^{22}$ Conceptually, selective deprotonation of nitriles with Grignard reagents is possible by rapidly forming an alkylmagnesium alkoxide ${ }^{23}$ in which coordination directs internal proton abstraction and geometrically prevents internal alkyl delivery to the electrophilic nitrile group $(\mathbf{9 a} \rightarrow \mathbf{1 0 a}$, Scheme 2). In practice, sequential addition of $i-\mathrm{PrMgBr}$ and methyl iodide to hydroxy nitrile $9 \mathrm{a}^{24}$ affords exclusively the axially methylated nitrile 9b-in direct contrast to the usual ${ }^{25}$ equatorial alkylation of $N$-lithiated cyclohexanecarbonitriles (compare with Scheme 1).

The remarkable installation of a 1,3-diaxial interaction, overriding typical steric preferences, 25 implies that stereoelectronic effects direct the retentive alkylation via the $C$-magnesiated nitrile 11a. Support for the intermediacy of $C$-magnesiated nitrile 11a stems from the stereodivergent methylation of the corresponding $N$-lithiated nitrile 12a where steric effects are the sole determinant of the alkylation stereochemistry. Deprotonating 9a with LDA, followed by addition of BuLi to prevent internal proton return, ${ }^{26}$ generates the $N$-lithiated nitrile 12a that alkylates methyl iodide exclusively ${ }^{27}$ from the sterically more accessible equatorial direction to afford 13b (Scheme 2). The stereodivergent alkylations of the $C$ - and $N$-metalated nitriles 11a and 12a stimulated a comprehensive series of alkylations of $C$ magnesiated nitriles with an array of electrophiles.

$C$-Magnesiated nitrile alkylations with alkyl halides. Rapid access to different $C$ magnesiated nitriles was conveniently achieved through sequential 1,2-1,4-Grignard additions 28 to 3-oxocyclohex-1-ene-1-carbonitrile (14,29 Scheme 3). Addition of methylmagnesium chloride to $\mathbf{1 4}$ generates the halomagnesium alkoxide $\mathbf{1 5}$ which exchanges $23 \mathrm{a}$ with a second Grignard reagent to afford $\mathbf{1 6}$ that, in turn, triggers conjugate addition $^{30}$ to the alkenenitrile functionality. The resulting bis-magnesiated nitrile $\mathbf{1 7}$ is 
conformationally destabilized by the two axial alkyl substituents, favoring equilibration to the $C$-magnesiated nitrile 11 (cf. 11a, Scheme 2).

Alkylating the $C$-magnesiated nitrile $\mathbf{1 1}$ with an array of electrophiles reveals a remarkable electrophile-dependent stereoselectivity (Table 1). ${ }^{19,31}$ Prior coordination between the electrophile and the magnesium atom of $\mathbf{1 1}$ does not appear to control the stereoselectivity 32 since alkylation with $\mathrm{Me}_{2} \mathrm{SO}_{4}$, an electrophile capable of metal-directed alkylation, ${ }^{33}$ affords exactly the same axially methylated nitrile $\mathbf{9 b}$ as that obtained by alkyating with MeI (Table 1, entries 1-2). Intercepting 11 with the more sterically demanding propyl iodide maintains the preference for axial alkylation despite the increased steric compression relative to alkylation with MeI (Table 1, entry 3). Alternatively, increasing the steric demand in the methylation by incorporating a phenyl group adjacent to the magnesiated nitrile-bearing carbon, still furnishes the axially methylated nitrile (Table 1 , entry 4 ).

Despite significant steric compression, the $C$-magnesiated nitrile $\mathbf{1 1}$ consistently intercepts alkyl halides and sulfonates with an exclusive preference for axial alkylation. Axial alkylation of the $C$-magnesiated nitrile $\mathbf{1 1}$ requires side-on overlap of the electrophilic $\sigma^{*}$ orbital with the large lobe of metal carbon $\sigma$ bond ${ }^{36}$ in a retentive, electrophilic substitution $-\mathrm{S}_{\mathrm{E}} 2_{\mathrm{Ret}}{ }^{37}$ (Scheme 4, 11a"). Although the side-on orbital overlap is far from optimal, the alternative co-linear approach of an $\mathrm{sp}^{3}$ hybridized electrophile to the small $\sigma$ lobe of the $\mathrm{C}-\mathrm{Mg}$ bond is sterically prohibitive (Scheme 4, 11a'). ${ }^{37}$ Reducing the side-on orbital overlap by alkylating 11a with $\mathrm{PrBr}$, having a smaller $\sigma^{*}$ orbital, is considerably more difficult ${ }^{38}$ than with PrI, leading to incomplete alkylation (13\% of $\mathbf{9 c}$ for $\mathrm{PrBr}$ compared to $70 \%$ for $\mathrm{PrI}$ ). The reactivity difference is not due to a competitive $\mathrm{E}_{2}$ elimination with $\mathrm{PrBr}$ since the addition of $\mathrm{D}_{2} \mathrm{O}$ results in high deuterium incorporation from the axial direction, analogous to preferential axial protonation upon addition of aqueous $\mathrm{NH}_{4} \mathrm{Cl}$ (Table 1, entry 5). ${ }^{39}$ Collectively, these comparative alkylations indicate that $C$-magnesiated nitriles require the more reactive alkyl iodides as electrophiles since incomplete alkylation occurs with the less reactive alkyl bromides.

The retentive alkylations of $C$-magnesiated nitriles with alkyl halide and sulfonate electrophiles contrast with the non-selective alkylations of $\mathbf{1 1}$ with allyl and benzyl bromide (Table 1, entries 6-8). Conceptually, the more reactive benzylic and allylic electrophiles, having larger $\sigma^{*}$ orbitals, might be able to access the small lobe of the Mg-C $\sigma$-bond for alkylation through an $\mathrm{S}_{\mathrm{E}} 2_{\text {(Inv) }}$ mechanism in addition to a side-on $\mathrm{S}_{\mathrm{E}} 2_{\text {(ret) }}$ alkylation (cf. 11a' and 11a", Scheme 4). Alternatively, single electron transfer (SET) from the electron rich $C$-magnesiated nitrile 11, formally a dianion, could generate a radical cation and an alkyl bromide radical anion. ${ }^{40}$ Subsequent bromide ejection from the radical anion and non-selective radical-radical recombination would account for the mixture of diastereomers.

Differentiating between these mechanistic scenarios was achieved through alkylations with cyclopropylmethyl iodide (Scheme 5). ${ }^{41}$ Cyclopropylmethyl iodide is a useful mechanistic probe: ${ }^{42}$ cyclopropylmethyl substitution provides evidence for an ionic $\mathrm{S}_{\mathrm{N}} 2$ displacement whereas butenyl alkylation, through single electron transfer, ring opening, and radical-radical recombination (18), indicates radical formation (Scheme 5). ${ }^{43}$ Experimentally, alkylating 11a with cyclopropylmethyl iodide affords approximately equal ratios of cyclopropylmethyl carbonitriles (9q and 13q) and but-3-enyl carbonitriles (9r and 13r), implying that a combination of electrophilic $\mathrm{S}_{\mathrm{E}} 2_{(\mathrm{Inv})}$ and $\mathrm{S}_{\mathrm{E}} 2_{\text {(Ret) }}$ alkylations compete with single electron transfer processes. ${ }^{44} \mathrm{~A}$ similar mechanistic scenario accounts for the diastereomers generated during alkylations with allyl and benzyl bromide (Table 1, entries 6-8).

C-Magnesiated nitrile alkylations with carbonyl electrophiles. Carbonyl electrophiles exhibit an extremely delicate stereoselectivity dependence in alkylations with the $C$ magnesiated nitrile 11 (Table 1, entries 9-15). ${ }^{45}$ Alkylations with phenyl isocyanate, 
cyclohexanone, and acetone proceed with exclusive retention of configuration (Table 1, entries 9-11) whereas cyclopropanecarboxaldehyde and cyclobutanone afford primarily the nitriles resulting from equatorial alkylation (Table 1, entries 12-13). ${ }^{46}$ In contrast, magnesiated nitrile 11a reacts with excess methyl cyanoformate and benzoyl cyanide to afford bis-acylated nitriles in which $C$-acylation occurs only from the equatorial direction (Table 1, entries 14 and 15).

A tentative explanation for the stereoselectivity differences exhibited in alkylations of $\mathbf{1 1}$ with carbonyl electrophiles emerges by comparing the reactivity of the $\pi^{*}$ orbitals. Particularly reactive carbonyl electrophiles, with large, diffuse $\pi^{*}$ orbitals, such as methyl cyanoformate and benzoyl cyanide, exert a sufficiently small steric demand to permit a co-linear approach to the small $\sigma$ lobe of the C-Mg bond (11a"', Figure 2). The co-linear trajectory, while more hindered than the side-on axial approach, benefits from a more favorable orbital overlap. Carbonyl electrophiles with a large steric demand, such as cyclohexanone, are unable to achieve sufficient proximity for overlap in a co-linear equatorial approach and preferentially alkylate by side-on overlap (11a"'", Figure 2).

The difference in the sizes of the $\pi^{*}$ orbitals effectively explains the rather perplexing 7:1 preference for the equatorial alkylation with cyclobutanone and the axial alkylation with acetone (Table 1, entries 13 and 11, respectively). The larger $\pi^{*}$ orbital of cyclobutanone, ${ }^{47}$ permits a more facile $\mathrm{S}_{\mathrm{E}} 2_{\text {(Inv) }}$ by more effectively interacting with the small $\sigma$ lobe in 11a"' than acetone which has a similar steric demand, but a smaller $\pi^{*}$ orbital and therefore preferentially alkylates only from the axial orientation. Acylations with methyl cyanoformate and benzoyl cyanide, particularly electron deficient electrophiles with large $\pi^{*}$ orbitals, similarly react through $\mathrm{S}_{\mathrm{E}} 2$ (Inv) processes. 45

\section{Conclusion}

Alkylations of $C$-magnesiated nitriles exhibit an unprecedented stereoselectivity dependence on the nature of the electrophile. $C$-Magnesiated nitriles efficiently alkylate alkyl iodides and sulfonates with retention of stereochemistry whereas acylations with aldehyde and acyl cyanide electrophiles occur with inversion of stereochemistry. Mechanistic probes indicate that the alkylations of $C$-magnesiated nitriles are primarily controlled by stereoelectronic effects, depending intimately on the size of the antibonding $\sigma^{*}$ or $\pi^{*}$ orbital. Allyl- and benzyl bromide and cyclopropylmethyl iodide are exceptions in which single electron transfer processes compete with substitution reactions.

The stereoelectronically controlled alkylations of $C$-magnesiated nitriles are stereochemically complementary to the sterically controlled alkylations of $N$-metalated nitriles. Synthetically, these two divergent strategies permit selective installation of diasteroemeric, quaternary centers from a single metalated nitrile.

\section{Experimental 48}

General Grignard addition-alkylation procedure: A THF solution of the Grignard reagent ( 2 equiv) was added to a $-78{ }^{\circ} \mathrm{C}$, THF solution $(0.1 \mathrm{M})$ of 3-oxocyclohex-1-ene-1-carbonitrile (14). ${ }^{29}$ After $1 \mathrm{~h}$ at $-78^{\circ} \mathrm{C}$, a THF solution of the second Grignard reagent (1.5 equiv) was added, or for reactions with only one Grignard reagent, 3.5 eq was added initially. The mixture was stirred at $-78{ }^{\circ} \mathrm{C}$ for $10 \mathrm{~min}$, and then warmed to room temperature. After $1.5 \mathrm{~h}$, the electrophile ( 3 equiv) was added neat, either at room temperature or with prior cooling to -78 ${ }^{\circ} \mathrm{C}$. Subsequent addition of saturated $\mathrm{NH}_{4} \mathrm{Cl}$ and extraction with EtOAc afforded a crude product that was washed with brine and dried $\left(\mathrm{MgSO}_{4}\right)$, concentrated, and purified by radial chromatography to afford the pure nitrile. 


\section{Supplementary Material}

Refer to Web version on PubMed Central for supplementary material.

\section{Acknowledgements}

Acknowledgment: Financial support of this research from NSF and, in part, NIH is gratefully acknowledged. Support for the x-ray facility from NSF (CRIF 0234872) is gratefully acknowledged as is assistance from Dr. Charles Campana in solving the $\mathrm{x}$-ray structure of $\mathbf{9 h}$.

\section{References}

1. (a) Fleming FF, Shook BC. Tetrahedron 2002;58:1.Arseniyadis, S.; Kyler, KS.; Watt, DS. Organic Reactions: Addition and Substitution Reactions of Nitrile-stabilized Carbanions. Willey; New York: 1984.

2. (a) Bradamante S, Pagani GA. J. Chem. Soc. Perkin Trans. II 1986:1035. (b) Dayal SK, Ehrenson S, Taft RW. J. Am. Chem. Soc 1972;94:9113.

3. Bug T, Mayr H. J. Am. Chem. Soc 2003;125:12980. [PubMed: 14558847]

4. Richard JP, Williams G, Gao J. J. Am. Chem. Soc 1999;121:715.

5. Bradamante S, Pagani GA. Adv. Carbanion Chem 1996;2:189.

6. Boche G. Angew. Chem. Int. Ed 1989;28:277.

7. Le Questel J-Y, Berthelot M, Laurence C. The bond length is the mean of CN distance obtained from 5059 nitriles in the Cambridge Structural Database. J. Phys. Org. Chem 2000;13:347.

8. (a) Sott R, Granander J, Hilmersson G. J. Am. Chem. Soc 2004;126:6798. [PubMed: 15161308] (b) Carlier PR, Lo CW-S. J. Am. Chem. Soc 2000;122:12819.Carlier PR, Lucht BL, Collum DB. J. Am. Chem. Soc 1994;116:11602.

9. Boche G, Harms K, Marsch M. The sole exception is a structurally biased lithiated cyclopropanecarbonitrile where $N$ - and $C$-coordination occur in a polymeric ladder structure. J. Am. Chem. Soc 1988;110:6925.

10. For computed structural differences in the extent of $C$ - and $N$-coordination for lithiated, sodiated and magnesiated acetonitrile see: Kaneti J, Schleyer PVR, Clark T, Kos AJ, Spitznagel GW, Andrade JG, Moffat JB. J. Am. Chem. Soc 1986;108:1481.

11. For $C$-metalated nitriles see: (a) Naota T, Tannna A, Kamuro S, Murahashi S-I. J. Am. Chem. Soc 2002;124:6842. [PubMed: 12059202] (b) Naota T, Tannna A, Murahashi S-I. J. Am. Chem. Soc 2000;122:2960. (c) Alburquerque PR, Pinhas AR, Krause Bauer JA. Inorg. Chim. Acta 2000;298:239. (d) Ruiz J, Rodríguez V, López G, Casabó J, Molins E, Miravitlles C. Organometallics 1999;18:1177. (e) Ragaini F, Porta F, Fumagalli A, Demartin F. Organometallics 1991;10:3785. (f) Porta F, Ragaini F, Cenini S, Demartin F. Organometallics 1990;9:929. (g) Ko JJ, Bockman TM, Kochi JK. Organometallics 1990;9:1833. (h) Cowan RL, Trogler W. J. Am. Chem. Soc 1989;111:4750. (i) Del Pra A, Forsellini E, Bombieri G, Michelin RA, Ros R. J. Chem. Soc. Dalton Trans 1979:1862. (j) Lenarda M, Pahor NB, Calligaris M, Graziani M, Randaccio L. J. Chem. Soc. Chem. Commun 1978:279. (k) Schlodder R, Ibers JA, Lenarda M, Graziani M. J. Am. Chem. Soc 1974;96:6893. (1) Yarrow DJ, Ibers JA, Lenarda M, Graziani M. J. Organomet. Chem 1974;70:133. For $N$-metalated nitriles see: (a) Tanabe Y, Seino H, Ishii Y, Hidai M. J. Am. Chem. Soc 2000;122:1690. (b) Murahashi S-I, Take K, Naota T, Takaya H. Synlett 2000:1016-1018. (c) Triki S, Pala JS, Decoster M, Molinié P, Toupet L. Angew. Chem. Int. Ed 1999;38:113. (d) Hirano M, Takenaka A, Mizuho Y, Hiraoka M, Komiya S. J. Chem. Soc. Dalton Trans 1999:3209. (e) Yates ML, Arif AM, Manson JL, Kalm BA, Burkhart BM, Miller JS. Inorg. Chem 1998;37:840. (f) Jäger L, Tretner C, Hartung H, Biedermann M. Chem. Ber 1997; 130:1007. (g) Zhao H, Heintz RA, Dunbar KR. J. Am. Chem. Soc 1996;118:12844. (h) Murahashi S-I, Naota T, Taki H, Mizuno M, Takaya H, Komiya S, Mizuho Y, Oyasato N, Hiraoka M, Hirano M, Fukuoka A. J. Am. Chem. Soc 1995;117:12436. (i) Hirano M, Ito Y, Hirai M, Fukuoka A, Komiya S. Chem. Lett 1993:2057. (j) Mizuho Y, Kasuga N, Komiya S. Chem. Lett 1991:2127. (k) Schlodder R, Ibers JA. Inorg. Chem. Soc 1974;13:2870. (1) Ricci JS, Ibers JA. J. Am. Chem. Soc 1971;93:2391.

12. For an analogous interconversion of ruthenium complexes see: Naota T, Tannna A, Murahashi S-I. J. Am. Chem. Soc 2000;122:2960. 
13. Kujime M, Hikichi S, Akita M. Organometallics 2001;20:4049.

14. (a) Fleming FF, Gudipati S, Zhang Z, Liu W, Steward OW. J. Org. Chem 2005;70:3845. [PubMed: 15876069] (b) Fleming FF, Zhang Z, Liu W, Knochel P. J. Org. Chem 2005;70:2200. [PubMed: 15760206] (c) Thibonnet J, Vu VA, Berillon L, Knochel P. Tetrahedron 2002;58:4787. (d) Thibonnet J, Knochel P. Tetrahedron Lett 2000;41:3319.

15. Orsini F. Synthesis 1985;500

16. Goasdoue N, Gaudemar M. J. Organometal. Chem 1972;39:17.

17. Bare TH, Hershey ND, House HO, Swain CG. J. Org. Chem 1972;37:997.

18. For a preliminary account see: Fleming FF, Zhang Z, Wei G, Steward OW. Org. Lett 2005;7:447. [PubMed: 15673261]

19. For an excellent discussion of electrophile-dependent alkylations of chiral organolithiums see: ClaydenJCh. 6. Organolithiums: Selectivity for Synthesis2002AmsterdamPergamon

20. Unpublished results with $\mathrm{BrMgN}-i_{-} \operatorname{Pr} 2$ and $\mathrm{BuMgN}-i_{-} \operatorname{Pr} 2$. For deprotonations with $\mathrm{BuMgN}-i_{-} \operatorname{Pr} 2$ see: Zhang M-X, Eaton PE. Angew. Chem. Int. Ed 2002;41:2169.

21. (a) Sumrell GJ. Org. Chem 1954;19:817. (b) Hauser CR, Humphlett WJ. J. Org. Chem 1950;15:359.For an exception see: Fauvarque J-F, Meklati B, Dearing C. C. R. Chim 1968;267:1162.

22. (a) Ivanov C, Markov P, Arnaudov M. Chem. Ber 1967;100:690.For the deprotonation of phenyl acetonitrile see: (b) Ivanov C, Markov P, Arnaudov M. Chem. Ber 1964;97:2987.

23. (a) Swiss KA, Liotta DC, Maryanoff CA. J. Am. Chem. Soc 1990;112:9393. (b) Turova NY, Turevskaya EP. J. Organomet. Chem 1972;42:9.

24. Cyclic nitrile 9a can be prepared through the conjugate addition of $\mathrm{MeMgCl}$ to 3-hydroxy-3methylcyclohex-1-enecarbonitrile ${ }^{\mathrm{a}}$ or, more conveniently, by sequential 1,2-1,4-addition to oxonitrile 14 (vide infra)Fleming FF, Wang Q, Zhang Z, Steward OW. J. Org. Chem 2002;67:5953. [PubMed: 12182627]

25. Fleming FF, Zhang Z. Tetrahedron 2005;61:747.

26. Zarges W, Marsch M, Harms K, Boche G. Angew. Chem. Int. Ed 1989;28:1392.Deprotonating 9a with LDA (2 equiv), followed by addition of MeI, affords a mixture of 9a, 13a, and 13b. Analogously, deprotonating 9a with $\mathrm{ClMgNEt}_{2}$ ( 2 equiv), followed by addition of MeI, afforded mainly the nitrile 9a with 13b as a minor component whereas deprotonating 9a with $\mathrm{ClMgNEt}_{2}$ (2 equiv), followed by the addition of $\mathrm{MeMgCl}$ ( 2 equiv) prior to the addition of $\mathrm{MeI}$, gave 13b (27\%), and $9 \mathbf{a}$ and 13a $(2.2: 1,28 \%)$ implying incomplete sequestration of the complexed $\mathrm{HNEt}_{2}$.

27. ${ }^{1} \mathrm{H}$ NMR analysis of the crude reaction mixture failed to identify any of the diastereomer $\mathbf{9 b}$.

28. Fleming FF, Zhang Z, Wang Q, Steward OW. Angew. Chem. Int. Ed 2004;43:1126.

29. Fleming FF, Zhang Z, Wei G. Synthesis 2005;3179

30. Fleming FF, Wang Q, Steward OW. J. Org. Chem 2003;68:4235. [PubMed: 12762721]

31. Electrophile dependent alkylations are relatively rare but often occur with chiral, tertiary benzylic and allylic organolithiums. ${ }^{\text {a }}$ Collectively, the occurance of electrophile-dependent stereoselectivity implies the intermediacy of a chiral organometalic intermediate, in this case the asymmetric $C$ magnesiated nitrile 11. Basu A, Thayumanavan S. Angew. Chem. Int. Ed 2002;41:717.

32. The Mg-O bond exhibits considerable back-bonding which dramatically reduces the Lewis acidity of the metal, making prior coordination with the electrophile unlikely: RicheyHGChapter 1. Grignard Reagents: New Developments20004Wiley, ChichesterNew York

33. (a) Meyers AI, Knaus G. J. Am. Chem. Soc 1974;96:6508. (b) Chassaing G, Lett R, Marquet A. Tetrahedron Lett 1978;471For an excellent discussion of the mechanism of this formally forbidden alkylations see: Hill, E.A., J. Organomet. Chem. 1975, 91, 123

34. Attempted oxidation of $9 \mathbf{k}$ afforded the cyclic ether $\mathbf{9 o}$, presumably by ionization of an activated cyclopropyl alcohol followed by internal cyclization: 


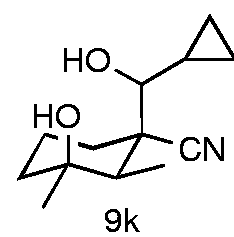

$(\mathrm{COCl})_{2}$, DMSO

$\mathrm{Et}_{3} \mathrm{~N}$

$9 \mathrm{k}$

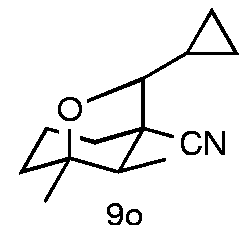

35. Bailey WF, Cioffi A. Magn. Reson. Chem 1987;25:181.

36. Carstens A, Hoppe D. Tetrahedron 1994;50:6097.

37. For an excellent overview of terms, steric constraints, and orbital overlap see: Gawley RE.

Tetrahedron Lett 1999;40:4297.

38. An analogous alkylation of 11a with 4-bromobutene similarly affords only $22 \%$ of the corresponding alkylated nitriles 9r and 13r with the protonated nitriles $9 \mathbf{a}$ and $\mathbf{1 3 b}$, predominating.
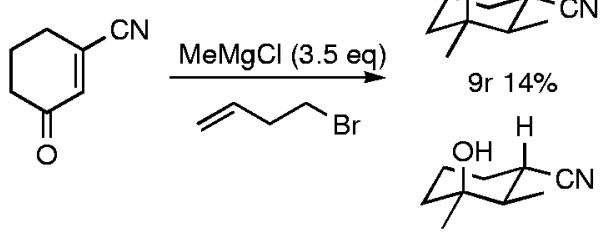

9 a $56 \%$

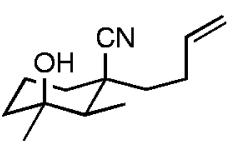

13 r $8 \%$

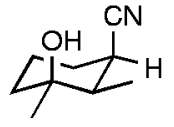

$13 b 8 \%$

39. The minor equatorial protonation isomer $\mathbf{1 3 b}$ may arise through a sterically accessible $S_{E} 2_{(I n v)}$ attack (cf. 11a')

40. For single electron transfer reactions of metalated nitriles see: (a) Werry J, Stamm H, Sommer A. Chem. Ber 1990;123:1553. (b) Roux-Schmitt M-C, Pettit A, Sevin A, Seyden-Penne J, Anh NT. Tetrahedron 1990;46:1263.For single electron transfer reactions of Grignard reagents see: (c) Chauffaille J, Hebert E, Welvart Z. J. Chem. Soc. Perkin Trans. II 1982:1645. (c) HillEARicheyHGJr.Chapter 1. Grignard Reagents: New Developments20004345WileyChichester

41. No alkylation of 11a is observed with the less reactive, but commercially available, cyclopropylmethyl bromide 
42. Gawley RE, Low E, Zhang Q, Harris R. J. Am. Chem. Soc 2000;122:3344.

43. Butenyl alkylation is, in some cases, possible through $\mathrm{S}_{\mathrm{N}} 2$ " alkylation: Alnajjar MS, Smith GF, Kuivila HG. J. Org. Chem 1984;49:1271.

44. Numerous alkylations of 11a with cyclopropylmethyl iodide identified a distinct mechanistic dependence on the quality of the $\mathrm{MeMgCl}$ employed in the 1,2-1,4-addition. In preliminary studies, ${ }^{18}$ use of low titre $\mathrm{MeMgCl}$ afforded almost exclusively the cyclopropylnitriles $\mathbf{9 q}$ and $\mathbf{1 3 q}$ (53\%) and only a trace of the butenylnitriles 9r and 13r. Presumably, the presence of alkoxide species causes a change in the nature of the reactive magnesiated nitrile which has minimal propensity toward single electron transfer

45. No alkylation was observed between 11a and DMF, methyl benzoate, or dimethyl carbonate

46. Control experiments in which the temperature was raised to rt cause equilibration of the intermediate alkoxy nitrile whereas quenching the reaction at $-78^{\circ}$ Cretains the stereochemical integrity of the first-formed alkoxy nitriles: Carlier PR, Lo CW-S, Lo MM-C, Wan NC, Williams ID. Org. Lett 2000;2:2443.For example, in the alkylation of 11a with cyclopropanecarboxaldehyde, elevating the reaction to ambient temperature causes an equilibration to a mixture of nitriles $9 \mathbf{k}$ and $\mathbf{1 3} \mathbf{k}$ and two diastereomeric lactones iii. Presumably higher temperatures facilitate not only retro-aldol fragmentation but also attack of the adjacent magnesium alkoxide onto the nitrile to generate ii which

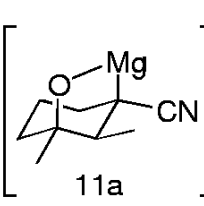

$11 \mathrm{a}$

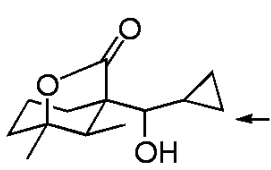

iii

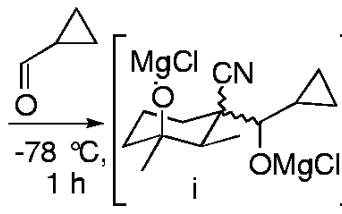

$\mathrm{rt}, 1 \mathrm{~h} ; \mathrm{NH}_{4} \mathrm{Cl}$

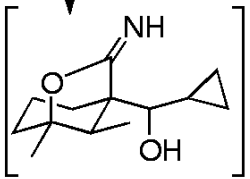

ii

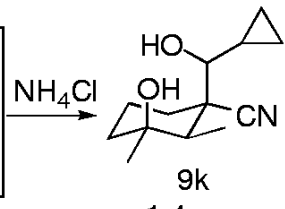

$1: 4$

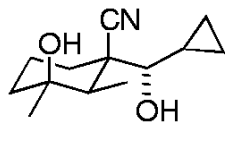

$13 k$

hydrolyzes to iii

47. An indication of the difference is provided by the stretching frequency of cyclobutanone 1775 $\mathrm{cm}^{-1}$ And acetone $1715 \mathrm{~cm}^{-1}$ : PretschESeiblJSimonWClercTTables of Spectral Data for Structure Determination of Organic Compounds1989I125Springer-VerlagBerlinsecond edition

48. For general experimental procedures see: Fleming FF, Hussain Z, Weaver D, Norman RE. J. Org. Chem 1997;62:1305. 

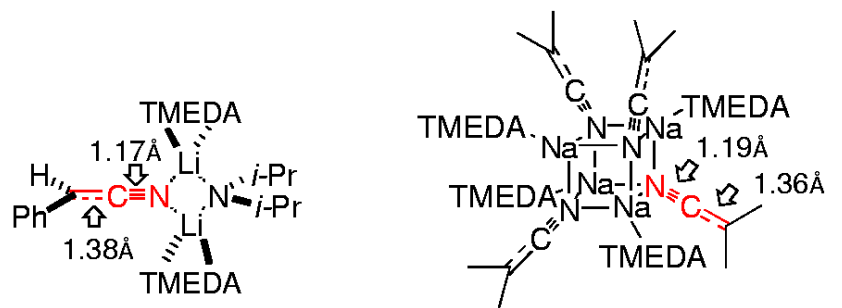

$1 \mathrm{~N}$-Metalated - planar

$2 \mathrm{~N}$-Metalated - pyramidalized
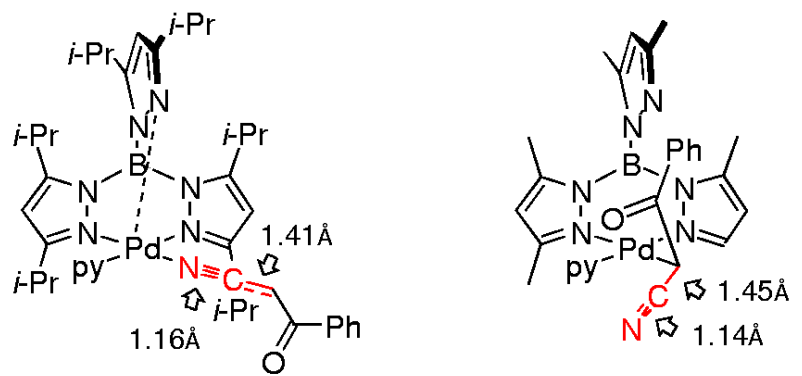

3 N-Metalated - planar

4 C-Metalated - pyramidal

Figure 1.

$\mathrm{X}$-Ray Structures of $N$ - and $C$-Metalated Nitriles. 

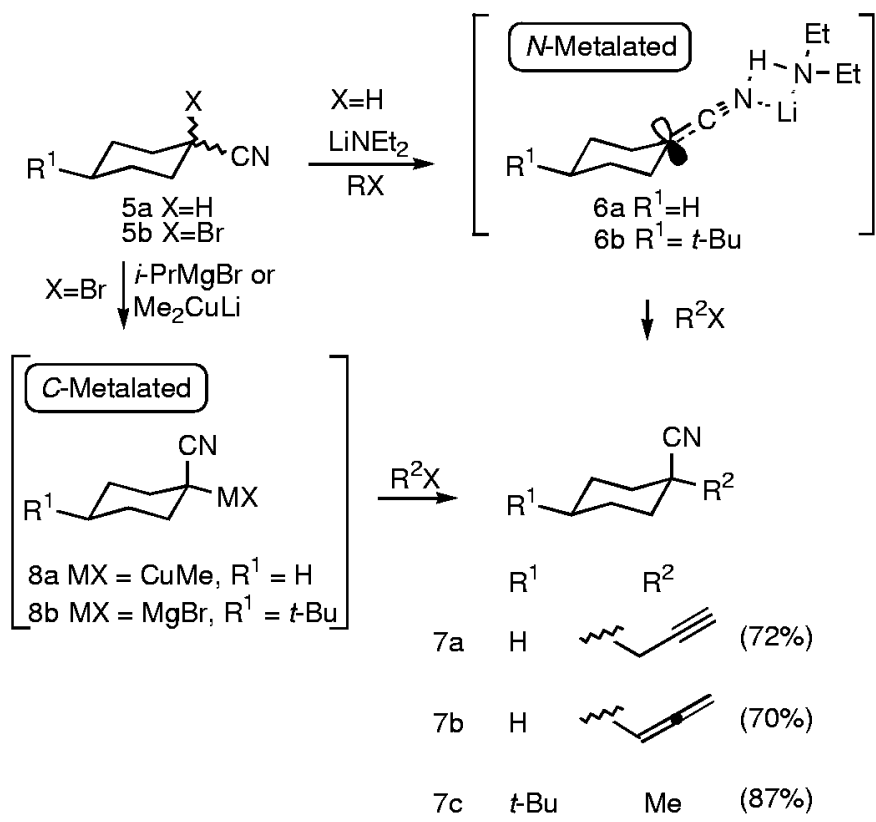

Scheme 1.

Divergent Regio- and Stereoselectivities of $N$ - and $C$-Metalated Nitriles 


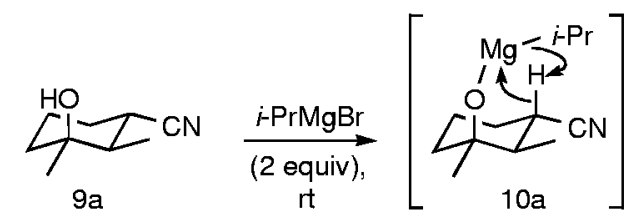

9a
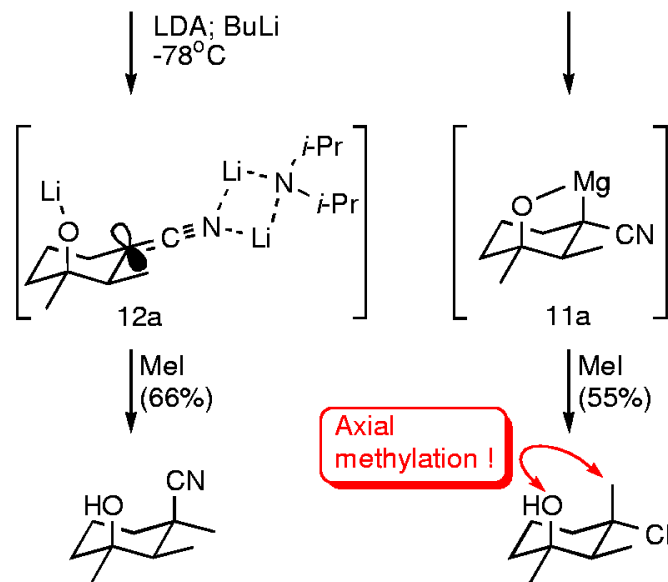

$13 b$

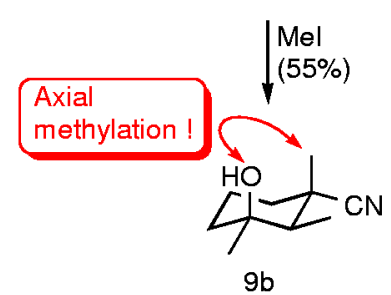

Scheme 2.

Stereodivergent Alkylations of $N$ - and $C$-Magnesiated Nitriles. 

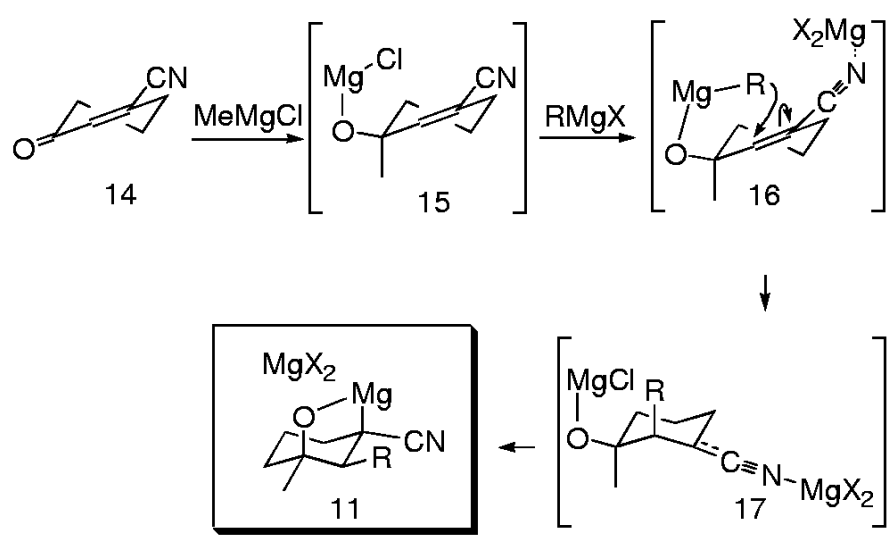

Scheme 3.

Sequential 1,2-1,4-Grignard Additions to Oxonitrile 14. 


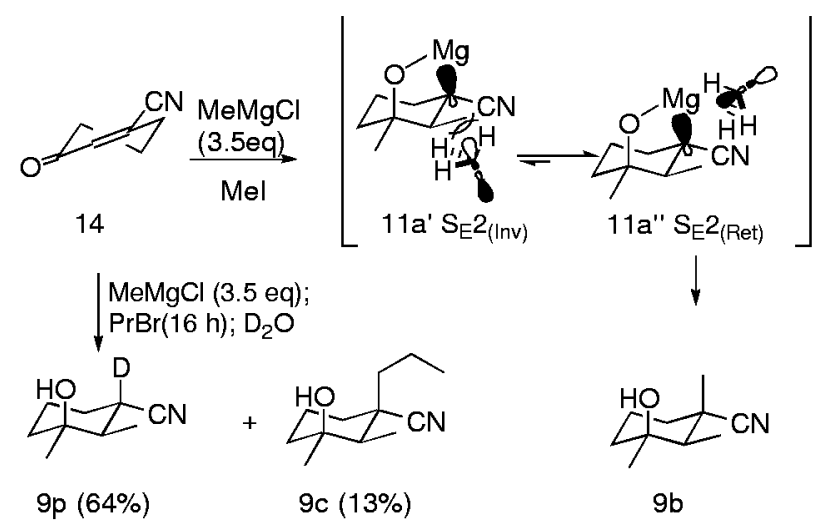

Scheme 4.

Stereoelectronic $\mathrm{S}_{\mathrm{E}} 2_{(\text {Ret })}$ Alkylations of Metalated Nitriles 


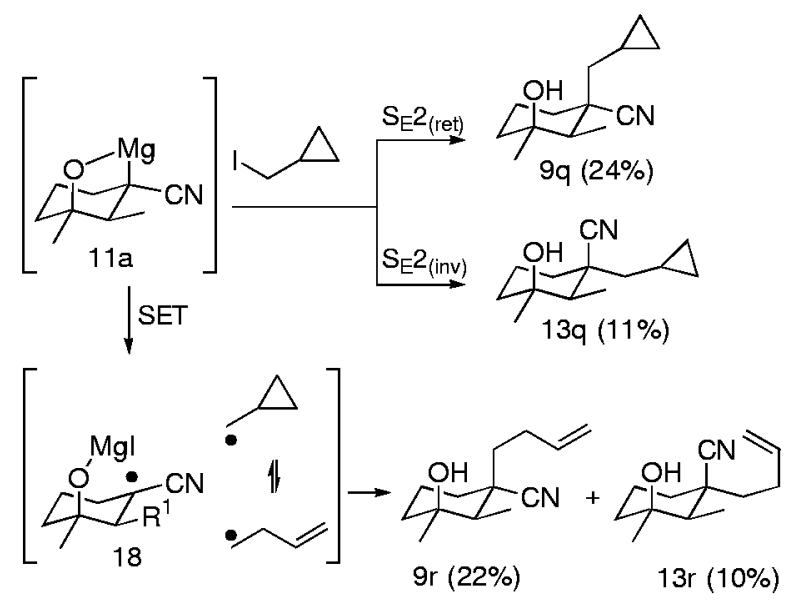

Scheme 5.

Mechanistic Probe for $C$-Metalated Nitrile Alkylations 

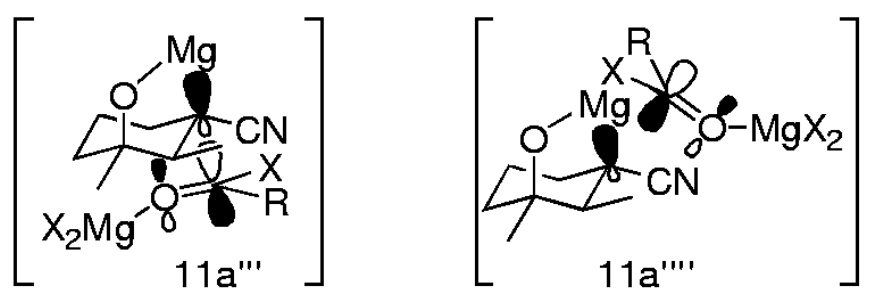

Figure 2.

Stereoelectronical control with carbonyl electrophiles. 
Table 1.

Electrophile-Dependent Alkylations of $C$-Magnesiated Nitriles

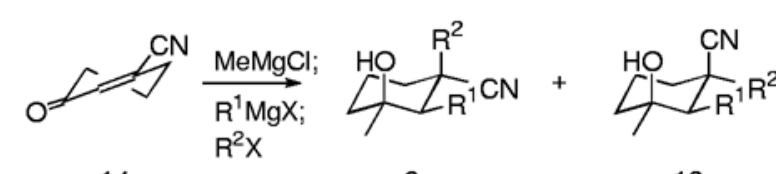

14

9

13

\begin{tabular}{|c|c|c|c|c|}
\hline Entry & $\begin{array}{c}\text { Grignard Reagent } \\
\text { R}^{1} \mathbf{M g X} ; \mathbf{R}^{2} \mathbf{M g X}\end{array}$ & Electrophile & Nitrile $^{a}$ & Yield $^{b}$ \\
\hline 1 & $\mathrm{MeMgCl}$ (excess) & Mel & & $86 \%$ \\
\hline 2 & $\mathrm{MeMgCl}$ (excess) & $\mathrm{Me} 2 \mathrm{SO} 4$ & & $57 \%$ \\
\hline 3 & $\mathrm{MeMgCl}$ (excess) & & & $70 \%$ \\
\hline 4 & $\mathrm{MeMgCl} ; \mathrm{PhMgCl}$ & Mel & & $71 \%$ \\
\hline 5 & $\mathrm{MeMgCl}$ (excess) & $\mathrm{NH}_{4} \mathrm{Cl}$ & 13 & $90 \%$ \\
\hline 6 & $\mathrm{MeMgCl}$ (excess) & & $13 e$ & $71 \%$ \\
\hline 7 & $\mathrm{MeMgCl}$ (excess) & $\mathrm{BnBr}$ & $13 f$ & $71 \%$ \\
\hline 8 & $\mathrm{MeMgCl} ; \mathrm{PhMgCl}$ & $\mathrm{BnBr}$ & & $61 \%$ \\
\hline
\end{tabular}

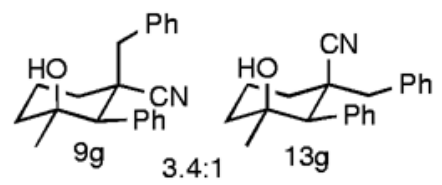




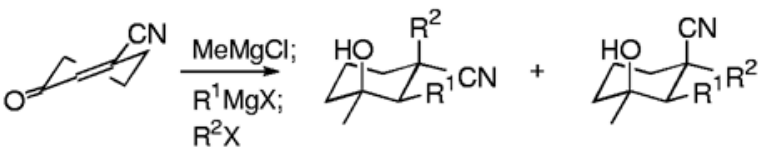

14

9

13

\begin{tabular}{|c|c|c|c|c|}
\hline Entry & $\begin{array}{c}\text { Grignard Reagent } \\
\text { R}^{1} \mathbf{M g X} ; \mathbf{R}^{2} \mathbf{M g X}\end{array}$ & Electrophile & Nitrile $^{a}$ & Yield $^{b}$ \\
\hline 9 & $\mathrm{MeMgCl}$ (excess) & $\mathrm{O}=\mathrm{C}=\mathrm{N}_{\mathrm{N}}$ & & $58 \%$ \\
\hline
\end{tabular}

$10 \quad \mathrm{MeMgCl}$ (excess)<smiles>O=C1CCCCC1</smiles><smiles>CC1(C)CCCC1(O)C1(O)CCCCC1(O)C#N</smiles>

$68 \%$

$11 \mathrm{MeMgCl}$ (excess)<smiles>[TeH]</smiles><smiles>CC(C)(O)C1(O)C2CCC(CC2)C1C#N</smiles>

$64 \%$

9j

$12 \mathrm{MeMgCl}$ (excess)<smiles>O=CC1CC1</smiles>

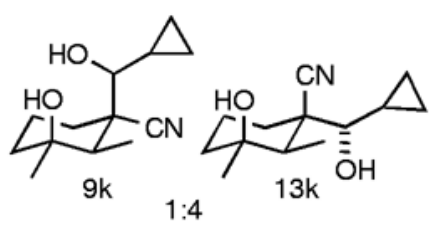

$55 \%^{c}$

13

$\mathrm{MeMgCl}$ (excess)<smiles>O=C1CCC1</smiles>

$59 \%{ }^{d}$

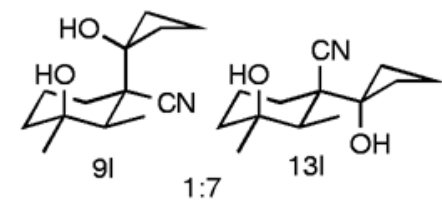

$14 \quad \mathrm{MeMgCl}$ (excess)<smiles>N#CC(=O)c1ccccc1</smiles>

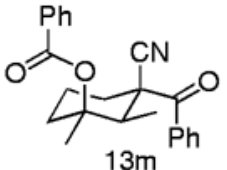

15 $\mathrm{MeMgCl}$ (excess)<smiles>COC(=O)C#N</smiles>

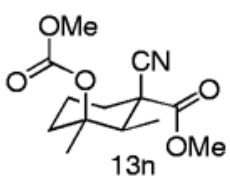

\footnotetext{
${ }^{a}$ Unless stated otherwise the stereochemical assignments are based on X-ray crystallographic analysis.

${ }^{b}$ Represents the overall 3-step yield for 1,2-addition, 1,4-addition, and alkylation.

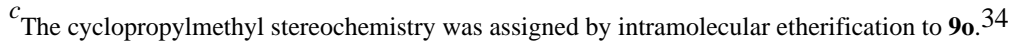


${ }^{d}$ Stereochemical assignments are based on the downfield shift of an equatorial nitrile carbon in ${ }^{13} \mathrm{C}$ NMR spectra. 35 